\title{
A.P.G.: An Intelligent Automatic Generator of Presentations for Tour-Guide Robots
}

\author{
J. Javier Rainer, Ramón Galán, Basil M. Al-Hadithi, Agustín Jiménez \\ Intelligent Control Group. Universidad Politécnica de Madrid \\ C/ José Gutiérrez de Abascal 2. 28006, Madrid, SPAIN \\ E-mail: javier.rainer@upm.es
}

Received 20 October 2010

Accepted 2 May 2011

\begin{abstract}
This work focuses on obtaining an automatic presentation generator for a robot guide. The most important aspect of this proposal is that the design uses learning as the means to optimize the quality of the presentations. This fuzzy system is used to select the most appropriate group of paragraphs for a presentation. The beliefs of the robot continue evolving in order to coincide with the opinions of the public. It uses a genetic algorithm for the evolution of the rules.
\end{abstract}

Keywords: Cognitive systems; learning; autonomous robot; fuzzy systems; decision making.

\section{Introduction}

Thanks to the numerous attempts that are being made to develop autonomous robots, increasingly intelligent and cognitive skills are allowed. Autonomous robots are intelligent machines capable of performing tasks in the world by themselves, without explicit human control over their actions Ref. 1.

Within the development of multiple applications for a mobile robot, probably one of the first real world applications of indoor service robots has been mobile robots serving as tour guides in museums or exhibitions. Such one is an extremely interesting application for researchers because allows them to advance in knowledge fields as autonomous navigation in dynamic environments, human robot interaction, cognitive skill, etc. We have developed our own interactive mobile robot called Urbano specially designed to be a tour guide in exhibitions Ref. 2. The basic characteristics of Urbano are described in section 3.
Our goal has been to create an automatic presentation generator that allows the flexible and dynamic display of information depending on the distinct kinds of audience and other parameters that characterize the presentation. The automatic selection of contents for composing sophisticated presentations is a non-trivial task. If the aims of the presentation, preference and interest for a particular subject have to be taken into account, it becomes even more complex.

The knowledge is based on ontology of domainspecific concept words. Ontologies have been known in computer science as consensual models of domains of discourse, usually implemented as formal definitions of the relevant conceptual entities, see Ref. 3 for more detail.

This paper is focused on the automatic generation of presentations by a robotic system. The MINERVA robot from Carnegie Mellon University was one of the first service robots for guiding tours. Others were Rhino, from Bonn University, or Xavier, built to participate in AAAI Robotic Competition in 1993. 
Some of the most recent works about automatic generation are described in Ref. 4-8. These works propose different architectures and methodologies than those presented here. They focus the problem of automatic generation of synchronized multimedia presentations as an answer to user queries. A number of them have used Rhetorical Structure Theory relations to link mixed-media resources into tree structures which can be translated into coherent multimedia presentations, Ref. 4.

In Ref. 6 Celentano et al. discuss the problem of defining schemas for such multimedia reports with a focus on the media coordination and synchronization constraints. In Ref. 9 Adali et al. present a process algebra for querying multimedia presentation databases. The algebra can be used to creating new presentations by retrieving different objects from existing ones or find presentations with specific properties.

What is original in our work is the introduction of fuzzy logic in the pruning of the resulting tree and in the quality index of the presentations. The presentation is represented by a tree, whose branches describe all possible presentation candidate sequences.

Other applications in mobile robotics for intelligent interaction with humans can be seen in Ref 10 . They discuss a probabilistic fuzzy rule-based life-long learning system, equipped with intention reading capability by learning human behavioral patterns, which is introduced as a solution in uncertain and time-varying situations.

The techniques used in A.P.G. are widely used as described in Ref.11, 12. The main contributions of Ref. 11 , is the illustration of the design and development of a fuzzy ontology based granular information retrieval system to improve the effectiveness of information retrieval decision making for various domains. In Ref. 12, presents a Modified Genetic Algorithm approach to obtain the optimal rule set and the membership function for a fuzzy classifier.

\section{Automatic Text Generation}

A.P.G. has access to a wide knowledge field of information as an automatic language generator. The history of natural language processing is characterized by the influence of artificial intelligent Ref. 13, 14. A natural language generator generally has access to a wide knowledge field from which it must select information to present to the users in various formats.
Generating text is, therefore, a decision-making problem with multiple restrictions: knowledge, available linguistic tools, the objectives of the user to whom the text will be directed, the situation, and past discourse. It deals with identifying the factors involved in this process and determining the factors that intervene in this process and its consequences Ref. 14-16.

An additional contribution of our work is to provide a dynamic framework that allows us to take into account each possible scenario. On the other hand, there can be no single general-purpose presentation format for all users, because each user differs in all aspects of interests and expertise levels, and in the devices used to visualize the requested information. These aspects are considered as restrictions.

As a result, to prepare a presentation, the candidate items can be multiple, depending on knowledge server. The items selected in the generation process contain information about the theme of the presentation, but must as well include the items that are semantically related to the presentation. Thus, for example, a discussion about the painter Diego Velazquez (15991660) will also speak about the Italian painter Michelangelo Caravaggio (1571-1610) due to his influence on Velazquez.

In our case a presentation generator is considered as a Cognitive Skill. It is assumed that skill development e.g. giving presentations progressively more suitable to the public being addressed - is a fundamental architectural epiphenomenon. Rather than viewing a presentation as a mere form of communication, the focus here is on leveraging it as a means of expanding socio-communicative skills.

Several research projects have been undertaken to develop software tools for generating narratives, histories or presentations, and they have described many characteristics for generating presentations, but the quality criteria vary, see Ref. 17.

The decision rules to establish the fact that a particular swap in the presentation strategy is useful and required are not clear yet. An interesting situation arises when the data changes and the environment are dynamic.

\subsection{Quality criteria of a presentation}

The quality of a presentation is defined by the different aspects that it characterizes, referred to in Ref. 18, as: nature, purpose, duration, and number of participants; 
for other authors Ref. 19, 20, such aspects as connecting with the audience, the interests of the participants, the first and last minute, changes of rhythm, and "being natural and having fun" are very important. All the aspects have in common the difficulty of being effectively quantified for a computer program.

The prototype as developed has proposed the following quality criteria:

- Differences in time used and the anticipated time

- Time dedicated to the theme

- Time dedicated to entertainment

- Difficulty in understanding the theme

- Interest in the theme

- Time dedicated to related information (anecdotal)

- Time for interaction with the public

- Original information

- Non-conventional focus

These criteria are also used to obtain the assessment of the public.

\section{Urbano}

We have developed our own interactive mobile robot called Urbano specially designed to be a tour guide in exhibitions. It has necessary cognitive skills for adaptation in the different environment like museums or events. Urbano possesses several features that could be used for interacting with the people (arms, face, voice synthesis, voice recognition, etc).

This section describes the Urbano robot system, its hardware /software and the experience we have obtained through its development and use until its actual mature stage.

\subsection{Urbano, an interactive Mobile Tour-Guide Robot}

Urbano robot hardware, see fig. 1, is a B21r platform from iRobot, equipped with a four wheeled synchrodrive locomotion system, a SICK LMS200 laser scanner mounted horizontally in the top used for navigation and SLAM, and a mechatronic face and a robotic arm used to express emotions as happiness, sadness, surprise or anger, thanks to its happiness model.

The robot is also equipped with two sonar rings and one infrared ring, which allows detecting obstacles at different heights that, can be used for obstacle avoidance and safety. The platform has also two onboard PCs and one touch screen.
The software is structured in several executable modules to allow a decoupled development by several teams of programmers, and they are connected via TCP/IP. Most of these modules are conceived as servers or services providers, as the face control, the arm control, the navigation systems voice synthesis and recognition, and the web server. The client-server paradigm is used, being the only client a central module that we call the Urbano Kernel. This kernel is responsible of managing the whole system, see Ref. 2.

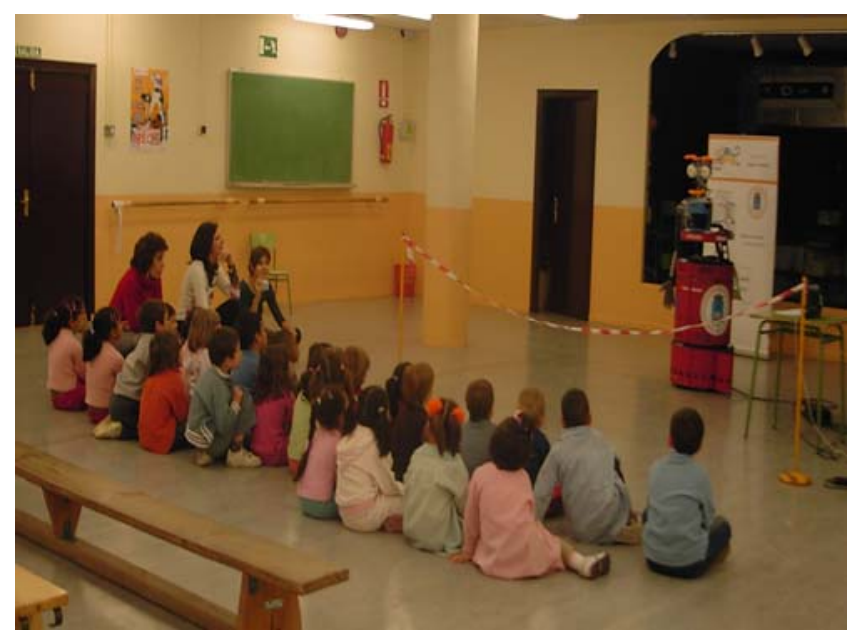

Fig. 1. Urbano Tour-Guide Robot.

URBANO robot has a technology based on distributed application software. The recent version is an agent based on architecture that uses a specific Common Object Request Broker Arquitecture (CORBA) approach as an integration tool. The robot has many functions: speaks, listens, navigates through the environment, moves his arm, responds to stimuli that affect its feelings.

\subsection{URBANOntology}

The knowledge server consists of a Java application developed using the libraries of Protégé-OWL application programming interface (API). The tool is capable of reading and editing files in ".owl” format where the knowledge is stored in the form of ontologies and the management of the information from the kernel is made by means of messages that codify the request of specific information, and the reply is obtained from the server or the introduction of new data.

The functions of the knowledge server are: loading and saving ontologies; creating, renaming, and deleting 
classes or instances; displaying properties of a class; showing subclasses or superclasses; showing or entering the value of a property; integrating one ontology into another; handling queries, etc.

\section{APG Agent}

APG agent software has been developed to be integrated in the architecture based on the agents that constitute the software of the Urbano robot. The developed computer application is activated on receiving a request from a user that selects a file that contains the pattern to be developed. Fig. 2 shows the A.P.G. architecture.

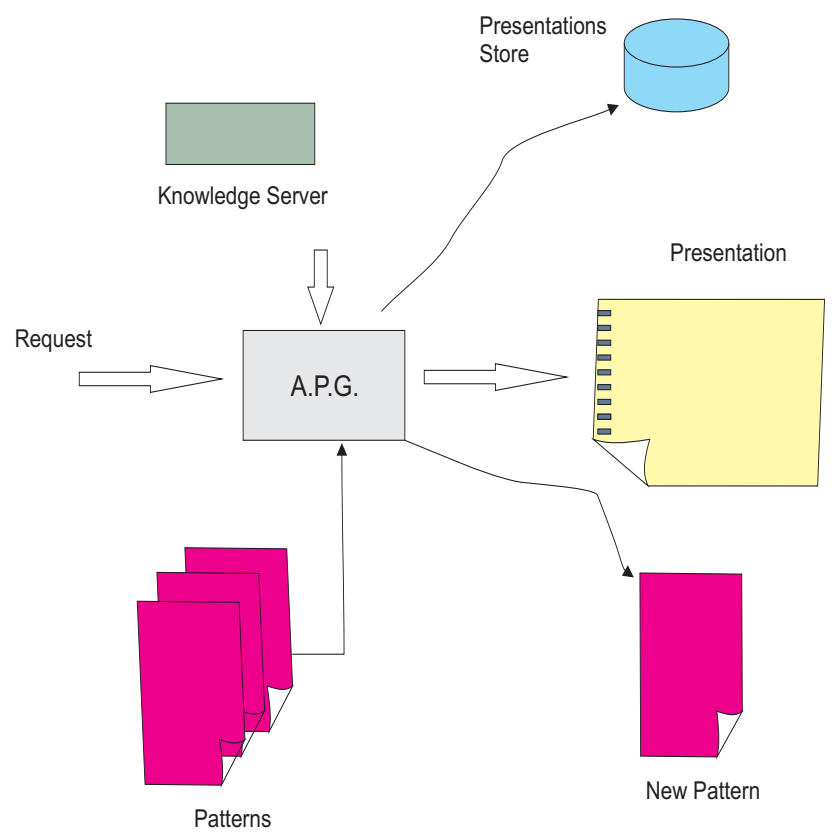

Fig. 2. A.P.G. Architecture.

APG will request all the information from the knowledge server agent, using restrictions that it needs to generate a file with the best presentation to be used by the system in the next performance by the robot.

Fig. 3 shows this architecture. The presentation is composed of paragraphs provided by knowledge server.

It must be mentioned the presentations have been stored with quality indices (QI) for each of them, and in some cases, when faced with a request very similar to a presentation already performed with high quality indices; this presentation will be used without needing to generate a new one.
The generator is a dynamic system where knowledge will increase and, therefore, it will improve the quality of the presentations. The robot will become increasingly better when making tour-guided visits. It also adds the ability to gesticulate while is conducting the presentation: it specifies the facial expressions and arm movements of the robot, the tone and type of voice to use, the position in the floor plan, and several other details for interaction with the public.

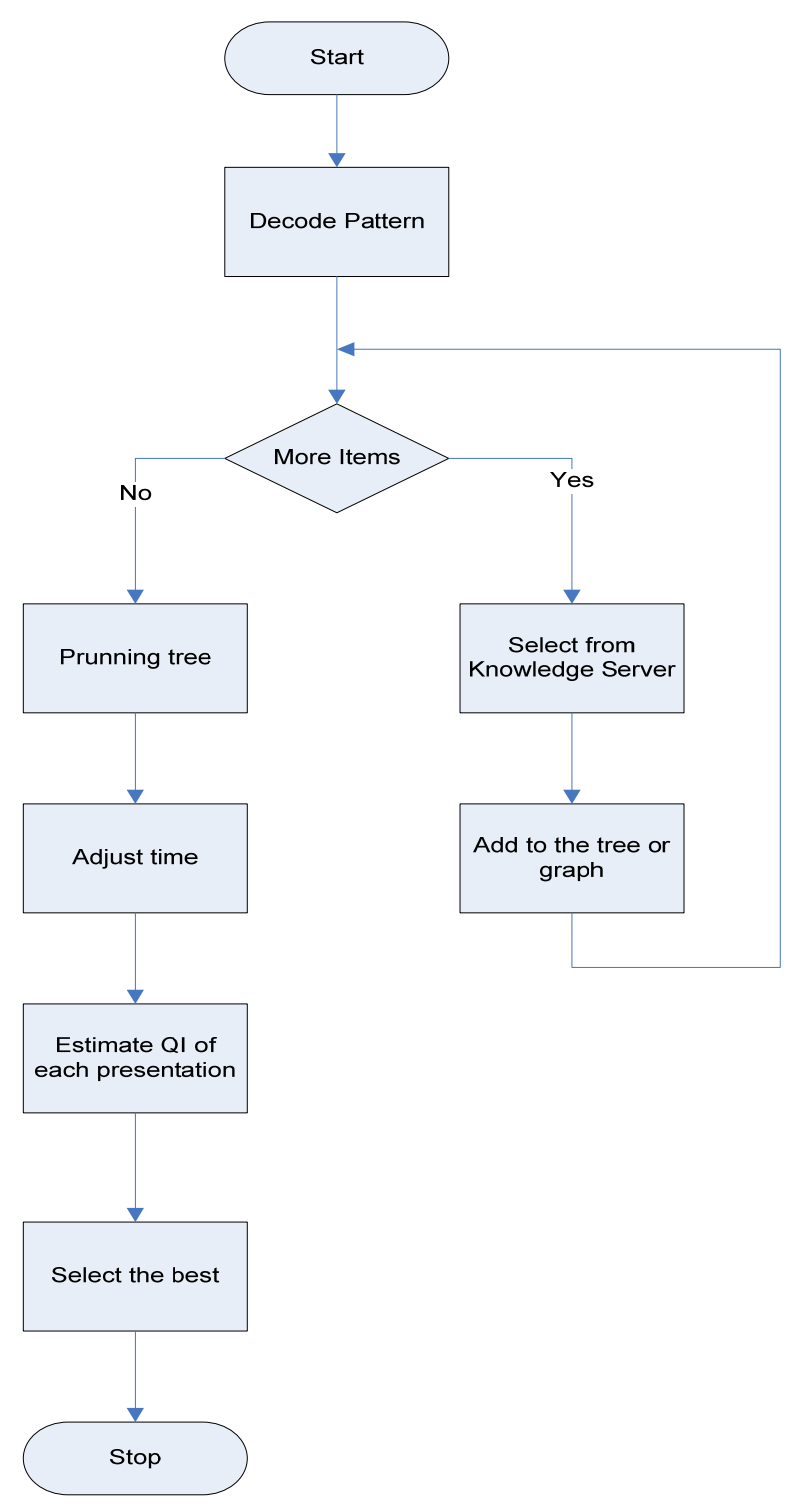

Fig.3. Flowchart of the proposed A.P.G. 


\section{Paragraphs}

The paragraph is used by the robot as the minimum element of expression. It is assumed that the paragraphs have a size such that coincidences or references between them lack meaning, and that they express a meaningful content. For example, the following paragraph introduces the painting "Las Meninas" by Diego Velazquez:

"A portrait of the infanta Margarita, daughter of Felipe IV (1605-1665), surrounded by her servants or 'family' in a hall of Madrid's Alcázar Palace.

This, the most famous of Velasquez's works, offers a complex composition built with admirable skill in the use of perspective, the depiction of light, and the representation of atmosphere." a

For each of the paragraphs there also exists a script that specifies the facial expressions and arm movements of the robot, the tone of voice and type of voice to use, the position in the floor plan, and various details for interaction with the public.

But in the ontology, each paragraph is "related" to the categories, which it belongs (e.g., in the previous paragraph: Is_a Painting, Is_a Description, Is_an Adult Level, Painted_by Diego Velazquez, etc. These relationships are used for the selection of the paragraph).

\section{Patterns and Trees}

The pattern, defined by the user, signals the elements that should form part of the presentation. For each item, these elements are established: its identification, its priority, its numerical order, its reference to the theme within available knowledge. There could be a limitation of time and a very large pattern, in that case the priority index indicates the most important content to be included, but there could be the opposite case, that there is time left, then the system incorporates content that it is not initially considered.

It uses XML as the language to represent the patterns, which guarantees an easy use with different tools and programming languages. XML has emerged as a de facto standard for encoding and sharing data among various applications. XML is also useful for structured information management, including information

\footnotetext{
${ }^{\text {a }}$ Paragraph extracted from online Gallery www.museodelprado.es
}

contained in knowledge server, see Ref. 21 for more detail. Our proposal is to design a software tool that helps the user to create and maintain the patterns.

APG requests from the knowledge server the available paragraphs for each theme, identified by its reference. The knowledge server will submit one or more paragraphs; because of a same concept can have several versions. Thus the pattern indicated in fig. 4 shows that first item of the presentation will be "museum_presentation" identified on the knowledge server as "Greetings" and it could involve, for example, three possible paragraphs, which will be included in the tree of possibilities, as is shown in fig. 5 .

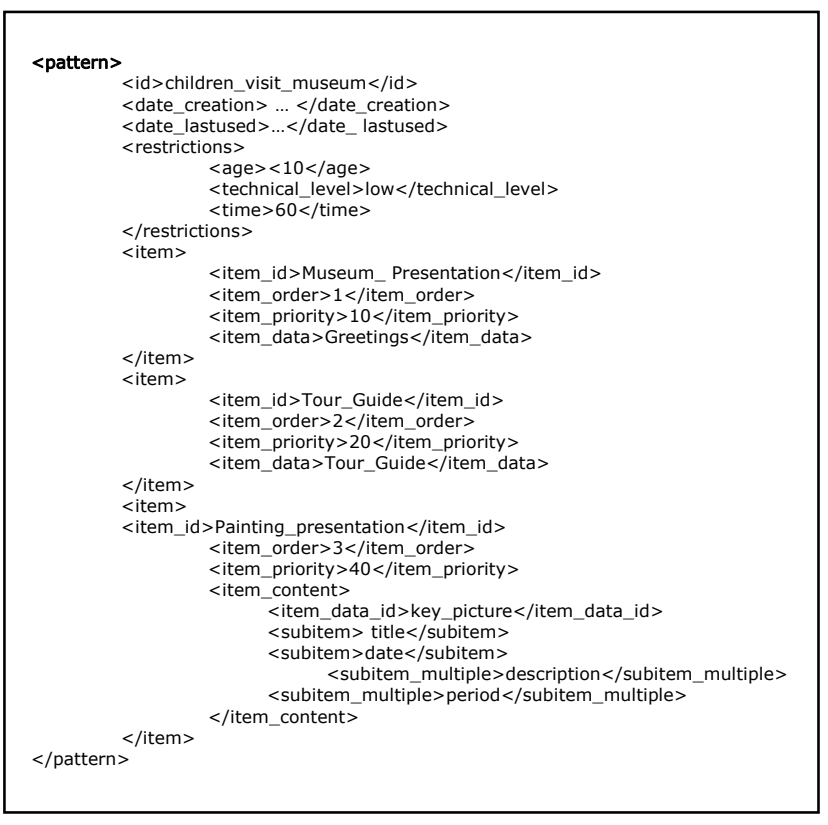

Fig.4. XML Pattern Example.

Some global restrictions including the level of audience will be used to prune the tree, eliminating the paragraphs that do not correspond to the requested level.

Three typical alternative heuristic searches have been tested to trim the tree. The first uses "brute force" to generate all the possible combinations and to group all the numeric values of the "quality criteria" of the paragraphs that form the presentation, and then, using a set of fuzzy rules, to estimate the quality index. It selects the presentation with the highest index. See fig. 6.

The second alternative uses "best-first search" so that as it goes along it takes the option that partially 
presents the best index. This alternative is undoubtedly without a doubt the fastest one, but it cannot guarantee the selection of the best option.

The third alternative modifies the previous one so that it generates "backtracking" if the quality index falls below a minimum.

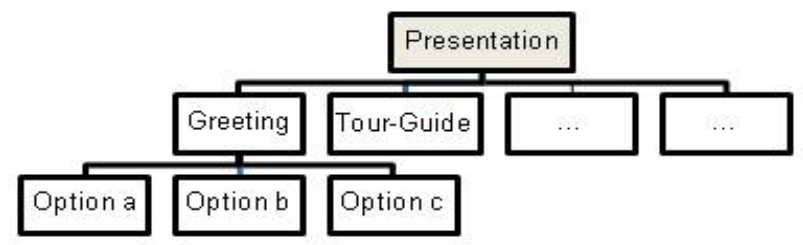

Fig. 5. Tree Data Structure

The presentation generated in this way analyzes the estimated time for its execution, and if this is greater than the anticipated one, it eliminates the paragraphs with the least necessary priority. On the other hand, if there is enough time, it includes some socially-oriented paragraphs such as jokes or comments about sports, politics, or local events.

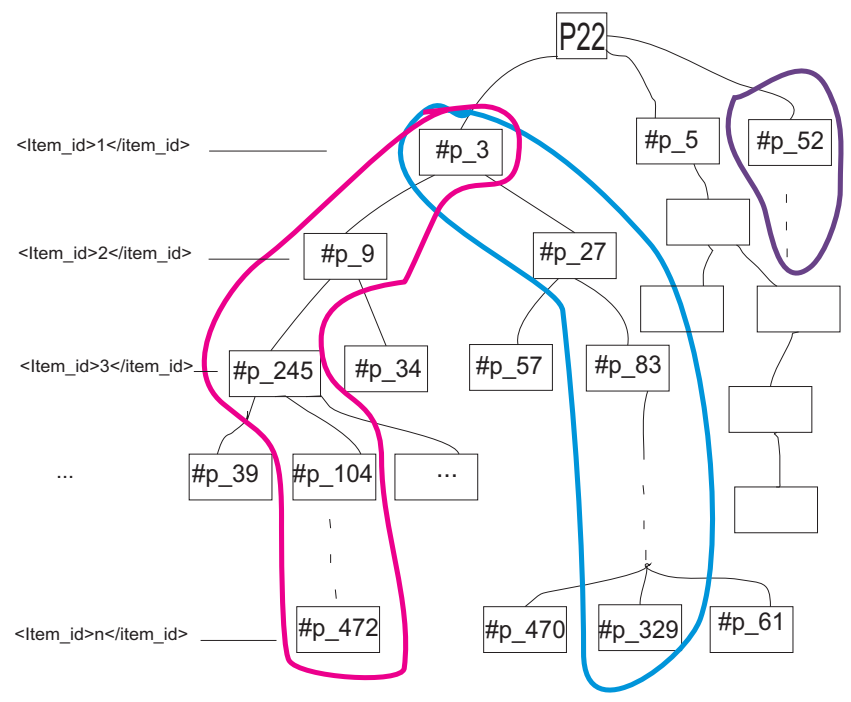

Fig. 6. Different options.

\section{Decisions-Making}

Decision-making is a part of the paradigm proposed by Zadeh, see Ref. 22 that has been currently examined in Ref. 23. In a dynamic scenario as ours, and because of the nature of the information that the system will handle, proper tools are needed to provide the intelligence for decision-making and supervision.
Decision-making is the cognitive process of selecting a course of action from multiple alternatives. Fuzzy set approaches to decision-making are usually most appropriate when human evaluations and the modeling of human knowledge are needed Ref. 24.

The proposed solution uses fuzzy rules to estimate the presentation quality. It will use some fuzzy variables that indicate some criteria to be considered in the presentation quality. The fuzzy system will select the best presentation.

The fuzzy rules offer more flexibility. These rules will be adjusted and expanded.

The rules look like:

$$
\begin{aligned}
& \text { If Criteria1 is LOW and } \\
& \text { Criteria2 is HIGH and } \\
& \ldots \\
& \text { CriteriaN is NORMAL then } \\
& \text { quality_index is NORMAL }
\end{aligned}
$$

All information available at the moment about the quality criteria and its influence on the quality index is stored in the ontology of the knowledge server.

The semantic network will indicate that the influence of time dedicated to the theme, expressed in a percentage, is VERY favorable if the time is VERY_HIGH but LOW favorable if the time is HIGH or NORMAL, and VERY unfavorable in any other case. This relationship is defined by belonging to specified categories. In the developed prototype, the fuzzy rules used to calculate the quality index are obtained by consulting the categories that belong to a criteria in the knowledge server.

Five linguistic terms are defined: VERY_HIGH (VH), HIGH (H), NORMAL (N), LOW (L), VERY_LOW (VL). The fuzzification phase uses the membership functions to initially equidistant triangles, but in the learning phase their centers can vary. The exit variable quality_index is also modeled with five terms and triangular functions. The technique of centroid method is used in the defuzzification phase.

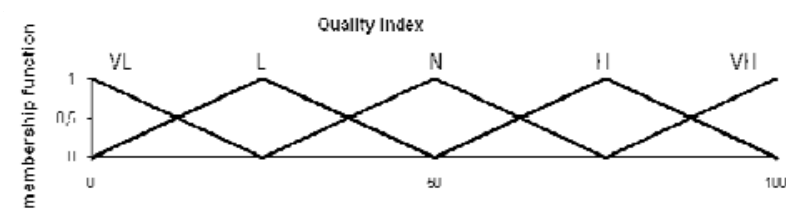

Fig. 7. Membership functions of the fuzzification / desfuzzification phase 
An interesting aspect is the partial quality estimation for the presentation. When considering three paragraphs (\#p1,\#p2,\#p3), for example, it may happen that some criteria have unrepresentative values; if, for example, \#p1,\#p2,\#p3 correspond to paragraphs of technical description, the entertainment criteria will be null and the presentation will have a low estimation. In the tests made using the best-first search, presentations are obtained with very poor estimations, and to compensate for this effect, an option of the best-first was introduced, but rejecting presentations with a low index.

If the categories of information expressed in the pattern are very generic, the number of possible presentations increases enormously, but it permits the robot to generate higher quality presentations.

\section{Learning Phase}

The most important characteristic of the proposal is the ability of the robot to learn. Initially it is thought that the robot will have a small number of quality criteria available to evaluate some presentations as good and others as bad, corresponding to the minimum level of education for a professional guide, in order to guarantee a minimum level of quality in its presentations.

A simple questionnaire has been designed so that the public can fill out after attending a presentation by the robot. It asks for an evaluation of each quality criteria known at the time, indicating whether the robot should spend more or less time on each item, and a percentage evaluation of what the public considers valuable in the presentation. The table I shows an example.

A proper statistical treatment of the questionnaires, eliminating the extremes and requiring a minimal quantity of data is performed.

Table 1. Quality Criteria.

\begin{tabular}{ll}
\hline Quality Criteria & Should be \\
\hline Time spent on the theme & $\mathbf{3 0}$ \\
Time spent on entertainment & $\mathbf{1 0}$ \\
$\begin{array}{l}\text { N. } \\
\text { New criteria to bear in mind }\end{array}$ & \\
Answers to questions & 2 \\
Global evaluation & $\mathbf{4 0 \%}$ \\
\hline
\end{tabular}

A genetic algorithm is used for an adjustment of linguistic terms and the membership functions, which will permit the quality index to be the closest to the average expressed by the public. To get a greater accuracy, the genetic algorithm is simultaneously used over several presentations as an attempt to offset the local minimal for a presentation. See fig.8.

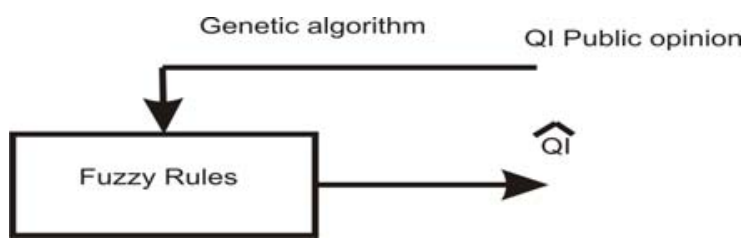

Fig. 8. Genetic algorithm adjusts the fuzzy rules.

The "time on the..." is used to eliminate individual cases generated by the genetic algorithm in which, while still generating a correct quality index, the evaluation of the criteria runs contrary to public opinion.

In the tests carried out, it was shown that the "beliefs of the robot" about the quality indices converge toward public opinion, and as a result generate presentations that are evaluated more favorably.

Learning is also presented on the criteria of paragraphs, which is performed by the recursive least square method. Each paragraph has some indices whose actual values are estimated from a sufficiently representative set of presentations where you know the real public opinion.

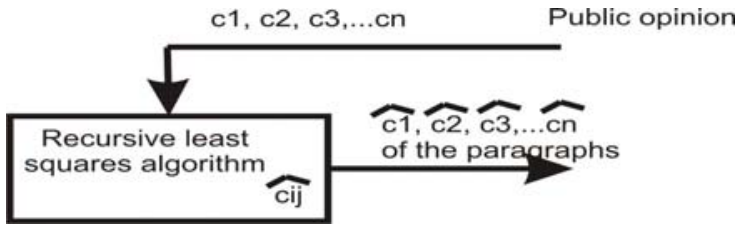

Fig. 9. Recursive least squares algorithm to change the criterias of the paragraphs

This will need a matrix A of NM * NC x NP * NC dimension, where:

$$
\begin{aligned}
& \mathrm{NP}=\text { number of paragraphs. } \\
& \mathrm{NC}=\text { number of criteria. } \\
& \mathrm{NM}=\text { number of presentations. }
\end{aligned}
$$

A vector $\mathrm{R}$ of $\mathrm{NP} * \mathrm{NCx} 1$ dimension has characteristics od two paragraphs

The public opinion is a vector $\mathrm{R}$ of $\mathrm{NP} * \mathrm{NCx} 1$ dimension. 
Thus, we will have $\mathrm{A} * \mathrm{R}=\mathrm{P}$, where $\mathrm{R}=\operatorname{pinv}(\mathrm{A}) * \mathrm{P}$. By applying the recursive least squares algorithm, the actual characteristics of paragraphs will be obtained.

\section{Experiments}

A $\mathrm{C}++$ based tool has been developed in order to implement both the fuzzy system and the genetic algorithms which are used for learning. Also, a standard MySQL database has been used for saving the presentations and the information that must be selected.

The prototype has allowed validating the efficiency of the system to generate appropriate presentations to the imposed requirements. Various criteria for pruning the tree of possible presentations have been tried out and it has been verified that the system is enough fast for its real-time working.

In connection with the learning, various experiments have been done in order to validate the feasibility of the method and to have an estimation of necessary time. Simulations have been performed with 5 linguistic terms and 4 variables or quality criteria; that means a total of 625 rules. It is used an archive file with 1300 presentations, whose quality is known for the public opinion. The genetic algorithm used has the following features: a genome composed by 625 variables, a population of 10 individuals, a crossing probability of 0.8 , and a mutation probability of 0.6 . This mutation probability, considerably higher than the usual 0.2 , has led to better results.

When there are no presentations covering all the possibilities in the archive file, some of the rules are never used, and the adjustment of quality index for these cases does not take place.

Table 2, shows other results with different number of quality criteria. The number of variables increase and the CPU spend considerable time.

Table 2. Experiments.

\begin{tabular}{cccc}
\hline $\begin{array}{c}\text { Quality } \\
\text { criteria }\end{array}$ & $\begin{array}{l}\text { Linguistic } \\
\text { terms }\end{array}$ & $\begin{array}{l}\text { Total } \\
\text { variables }\end{array}$ & CPU time \\
\hline 3 & 5 & 125 & 1 hours \\
4 & 5 & 625 & 8 hours \\
7 & 5 & 78125 & 14 hours \\
\hline
\end{tabular}

As a future work, it is proposed to analyze the effect of introducing, in real-time, new criteria which affect the quality index, and to analyze, if it is possible, the learning of features of quality for each available paragraph.

Matlab tool has been used to demonstrate that the least squares method ensures the learning of the system about the criteria of paragraphs. The experiments were performed with 40 paragraphs, 5 criteria or characteristic of a paragraph and of 500 sets of presentations, which results in satisfactory results in real time.

Other experiments have been implemented. More than 150 presentations are simulated. Each presentation consists of 1 to 40 paragraphs. The assessment criteria of each paragraph are generated randomly. It has been considered three different values for the probability of including a paragraph in a presentation (5\% represented in green, $10 \%$ in red, $25 \%$ in blue). To measure the quality of learning, it has been taken into consideration the percentage of erroneous assessments of the criteria of the 40 paragraphs.

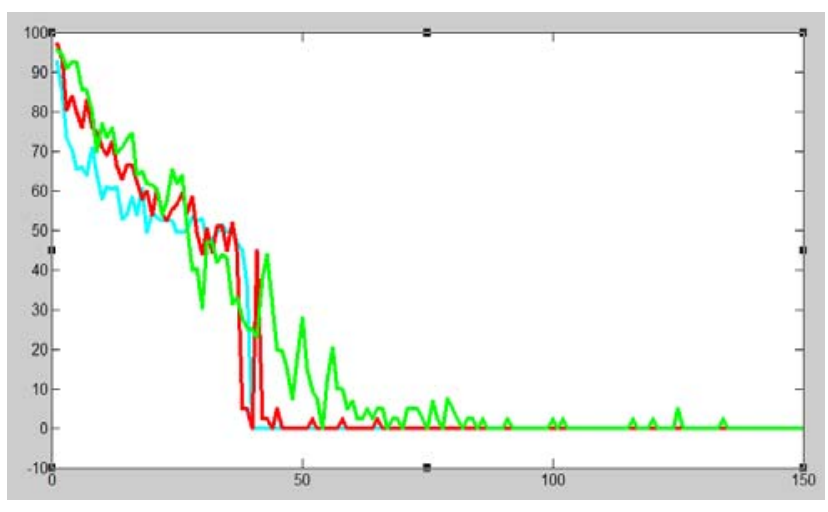

Fig. 10. Evolution of assessments based on the number of presentations.

As it can be seen in fig. 10 that as more presentations have been realized and more public opinion has been taken as well, the erroneous assessments is reduced.

\section{Conclusion}

As a conclusion, an automatic generator of presentations has been analyzed, special attention has been given to a mechanism for automatic generation of presentations, analysis of search algorithms, learning phase and optimization of fuzzy logic rules, taking into account 
the intrinsic difficulty of natural language processing and automatic generation.

This development is appropriate for very realistic generation of presentations, and it will allow tour-guide robots to offer more affective learning and a dynamic tour-guide visit, because the public generally has become more sophisticated and, also, its expectations and demands. It provides a better use of knowledge management. At the same time, advances in a new visitor-oriented approach, progress towards the creation of modular and scalable scenarios.

It presents a novel approach to the use of a computer ontology to represent the corpus that the robot works with, and the quality criteria for a presentation.

Methodology designed for the automatic generation of presentations can be used for other applications related with decision making for autonomous robots. In the Urbano project design; there is a scheduler agent that selects the best task between different tasks, which the system has to do in a day. The criteria used for selecting the adequate task in short-term plan can be revised to optimize the long-term objective. A happiness model, as long-term objective, and a decision-taking mechanism, as short-term planner, was modeled for Urbano. Both use fuzzy logic and are adjusted by genetic algorithms that use the public opinion to learn to be a good tour-guide robot.

These models of learning represent a significant step in the evolution of learning machines. The two form of learning have been evaluated. Results show that genetic algorithm and least-squares method allow the development and learning system.

\section{Acknowledgements}

This work is founded by the Spanish Ministry of Science and Innovation (ROBONAUTA: DPI 200766846-C02-01 and ARABOT: DPI 2010-21247-C0201) and supervised by CACSA whose kindness we gratefully acknowledge.

\section{References}

1. G. Bekey, Autonomous Robots: from biological inspiration to implementation and control, MIT Press books, 2005.

2. D. Rodríguez-Losada, F. Matia, R. Galán, M. Hernando, J. M. Montero, and J. M. Lucas, Urbano, an Interactive Mobile Tour-Guide Robot. Advances in Service Robotics. Ed. H. Seok. In-Teh, 2008, pp 229-252.
3. M. Uschold and M. Grüninger, "Ontologies: Principles, Methods, and Applications,” Knowledge Eng. Rev., vol. 11, 1996, pp. 93-155.

4. E. André. And T. Rist, "Generating Coherent Presentations Employing Textual and Visual Material. In Artificial Intelligente Review, Special Volume on the Integration of Natura1 Language and Vision Processing, 9(2-3):147-165, 1995.

5. P.M. Barnaghi and S.A. Kareem, "Ontology-Based Multimedia Presentation Generation,” IEEE TenCon, 2005.

6. A. Celentano and O. Gaggi, "Schema Modelling for Automatic Generation of Multimedia Presentations," Proc. 14th Int. Conf. on Software engineering and knowledge engineering, Italy, 593-600, 2002.

7. F. Frasincar, G. Houben, P. Barna, and C. Pau, "RDF/XML-based Automatic Generation of Adaptable Hypermedia Presentations,” Proc. Int. Conf. on Information Technology: Computers and Communications IEEE, 2003.

8. S. Little, J. Geurts and J. Hunter, "Dynamic Generation of Intelligent Multimedia Presentations though Semantic Inferencing," The 6th European Conference on Research and Advance Technology for Digital Libraries, ECDL 2002, LNCS 2458, pp. 158-175. Italy, 2002.

9. S. Adali, M. L. Sapino, and V. S. Subrahmanian. An algebra for creating and querying multimedia presentations. Multimedia Systems 8(3): 212-230, 2000.

10. Z. Z. Bien, H. Lee, J. H. Do, et al. Intelligent Interaction For Human-Friendly Service Robot In Smart House Environment. International Journal of Computational Intelligence Systems, Vol. 1, No. 1 (January, 2008) 7793. Atlantis Press.

11. R.Y.K. Lau, C.C.L. Lai, Y. Li, Fuzzy Ontology Mining and Semantic Information Granulation for Effective Information Retrieval Decision Making. International Journal of Computational Intelligence Systems, Vol.4, No. 1 (February, 2011). Atlantis Press.

12. P.Ganesh Kumar, Fuzzy Classifier Design using Modified Genetic Algorithm. International Journal of Computational Intelligence Systems, Vol.3, No. 3 (Sep, 2010), 334-342. Atlantis Press.

13. R. Cole , J. Mariani , H. Uszkoreit, A. Zaenen, and V. Zue, Survey of the State of the Art in Human Language Technology. Cambridge: Cambridge University Press, 1996.

14. K. Sparck Jones, Natural languages processing: a historical review. University of Cambridge, 2001.

15. J. Allen, Natural Language Understanding, Redwood City, Ca.: Benjamin /Cummings, $2^{a}$ ed., 1995.

16. D. Jurafsky, and J. Martin, Speech and Language Processing. Prentice-Hall, 2000.

17. M. Cavazza, F. Charles, and S. J. Mead, "Characterbased interactive storytelling,” IEEE Intelligent Systems: Special Issue on AI in Interactive Entertainment, 17(4):17-24, 2002.

18. F. Laure, Técnicas de Presentación. Cecsa, 2002. 
19. S. Berkun, Confessions of a Public Speaker. O’Really, 2010.

20. J. Weissman, Presenting to Win: The Art of Telling Your Story. Pearson Education, 2009.

21. M. Y. Maarouf and S. M. Chung, "XML Integrated Environment for Service-Oriented Data Management," 20th IEEE International Conference on Tools with Artificial Intelligence, 2008.

22. L. A. Zadeh, "Fuzzy sets," Information and Control, 8, 338-353, 1965.

23. L. A. Zadeh, "Is there a need for fuzzy logic?" Information Sciences, 178(13), 2751-2779, 2008.

24. C. Kahraman, "Fuzzy set applications in industrial engineering”, Information Science, Volume 177, Issue 7, Pages 1531-15321, 2007. 